\title{
Kinetic analysis of endothermic degradation of magnesium hydroxide, calcium hydroxide and calcium carbonate in the context of passive fire protection
}

\author{
Aleix Ciudad*1, L. Haurie ${ }^{2}$, and A.M. Lacasta ${ }^{1}$ \\ ${ }^{1}$ Departament de Física Aplicada, Universitat Politècnica de \\ Catalunya, Av.Dr. Marañón 44, E-08028 Barcelona, Spain \\ ${ }^{2}$ Departament de Construccions Arquitectòniques II, \\ Universitat Politècnica de Catalunya, Av. Dr. Marañón 44, \\ E-08028 Barcelona, Spain
}

September 17, 2013

\begin{abstract}
In a fire scenario, huge amounts of heat are generated and high temperatures rapidly achieved in such a way that the integrity of structural materials becomes compromised. One of the aims of Passive Fire Protection is the use of building materials that are able to absorb at least part of that heat and maintain the structural materials under critical temperatures for longer times in order to gain evacuation time. Gypsum panels are commonly used in building walls, but they only absorb heat at temperatures around $110^{\circ} \mathrm{C}$. We use three inorganic fillers, $\mathrm{Mg}(\mathrm{OH})_{2}, \mathrm{Ca}(\mathrm{OH})_{2}$ and $\mathrm{CaCO}_{3}$, which undergo endothermic transitions at high temperatures to obtain an improved panel with a richer heat absorbing profile [1]. With this formulation, the time to
\end{abstract}

*aleix.ciudad@upc.edu 
reach temperatures of the order of $500^{\circ} \mathrm{C}$, critical for steel and reinforced concrete, is significantly increased. In this work we focus on the kinetics of the endothermic fillers as an essential ingredient for further spatially extended simulations that include macroscopic heat and mass transfer phenomena or sample heterogeneities. However, kinetics may be affected as well by heat transfer effects that occur at molecular levels. Keywords: Passive Fire Protection, endothermic reactions, heating rate, thermogravimetry, self cooling

\section{Introduction}

In Fire Safety research there are two related but different approaches to the protection against fire in a building. On the one hand there is Active Fire Protection (AFP) where fire has to be detected and a later action has to be performed, like the activation of sprinklers. On the other hand we have Passive Fire Protection (PFP), where fire hazards are reduced by processes undergone by specialized materials. These processes do not trigger further responses. Typical examples are cable coating or firewalls. In this work we focus our attention to a specific aspect of PFP: the heat absorption of improved drywalls. The use of drywalls, also called wallboards, is widespread in building construction, so it can be used as a PFP material $[2,3,4,5,6,7]$. However, usual drywalls are gypsum panels which absorb heat only at gypsum dehydration temperatures $\left(100-110^{\circ} \mathrm{C}\right)$. In a previous work [1] we developed an improved panel where three alkaline earth fillers, $\mathrm{Mg}(\mathrm{OH})_{2}, \mathrm{Ca}(\mathrm{OH})_{2}$ and $\mathrm{CaCO}_{3}$, are added to gypsum. These three materials have decomposition temperatures around 400, 500 and $850^{\circ} \mathrm{C}$, respectively. Consequently, the resulting compound has a rich heat absorbing profile that works in a wide temperature domain. When exposed to high temperature situations, samples made of our compound are able to keep temperatures under critical regimes for longer times than gypsum-made panels without additions that contribute with endothermic reactions. [2, 8, 9, 10, 11, 12, 13, 14, 15].

The improvement of drywalls by the use of fillers and the role of porosity and heterogeneities is an open and promising field where the numerical simulation of spatially extended systems is an essential tool for predicting and understanding the behavior of the tested materials. However, prior to a spatial numerical simulation, kinetic information of the components is re- 
quired in order to compute the local and instantaneous reaction rate, i.e. the velocity of reaction at each position and time. The usual approach is to make use of calorimetric data to extract kinetic parameters that are used as an input in the simulations. Under this scheme, heat and mass transfer effects depend on the underlying kinetics that we use. But such effects are rarely incorporated as ingredients of the kinetic model. This influence would had no meaning if numerical simulations had such a fine grid that the spatial cell size would be small compared with the size of heat and mass transfer effects. However, our main hypothesis is that the endothermic reaction and its subsequent heat absorption produces a self-cooling effect in a very small size scale. No grid can achieve such a size and this effect has to be included in the kinetic equations in order to take this phenomenon into account.

Usual calorimetric techniques to characterize the kinetic parameters are Thermogravimetry (TG), Differential Thermal Analysis (DTA) or Differential scanning calorimetry (DSC) [16]. In this work we will make use of TG data under different heating rates in order to explore the possibilities of our proposed model. Other works have already dealt with endothermic kinetics, either focusing on calcium carbonate [17, 18, 19, 20, 21, 22], Magnesium hydroxide $[23,24,25]$ or Calcium hydroxide [26]. There are many approaches to solid state kinetics. Mainly, three rate limiting mechanisms are proposed: diffusion, chemical reactions or nucleation. There is a wide variety of different specific kinetic models for each mechanism. For the case of chemical reaction models, different conversion functions, one for each model, are tested. Sometimes, isoconversional analysis is applied to provide a phenomenological characterization of the underlying processses. Conventional models usually fit the data with lower accuracy than the isoconversional approach, but they contain a reduced set of parameters, while isoconversional analysis, even though it usually gives better fits, needs a different parameter for every conversion value. The usual idea, when working with models, is to test all the available functions and decide which one fits the data with higher accuracy. However, sometimes different models fit the data with similar results, so even if a bad fit can discard a model, a good one cannot validate it as the true mechanism. Our approach is somewhat different because instead of claiming that our model is the actual mechanism we try to show that, even if there are other factors that can influence the kinetics, the self-cooling effect cannot be neglected because even a very small self-cooling has a strong overall effect. Having said that, we fit the data by considering a simple one-step re- 
action mechanism with a self-cooling correlation, showing that this is enough for a first order description with a reduced set of parameters and without overcomplicating the mathematical expressions of the model.

One of the most interesting aspects is the finding that the peaks in the signals are asymmetric [27]. Such asymmetry is a trace of a correlation mechanism. There are other models that are also able to fit the data as they are introducing correlating behavior in a phenomenological way. For example, the so-called order of reaction $n$, when it is different from one, produces a correlation kinetics, but the microscopic meaning of this correlation remains unclear. In our model, the correlation parameter will have a precise microscopic meaning. Moreover, our model will allow some analytical results that will make the fit process easier. In short, we propose that self-cooling due to heat absorption is one of the mechanisms that produce a correlated kinetics and, consequently, it has to be incorporated into the kinetic model in order to shed some light on the actual heat exchanges that occur in a material when fire heats it up.

\section{Theoretical model}

An endothermic reaction has a positive value of enthalpy. This implies that the entropic term is the responsible for making the overall reaction spontaneous, i.e. with negative Gibbs free energy difference $\Delta \mathrm{G}$. At room temperature, none of the three inorganic solids of our compound decompose spontaneously. They need higher thermal energies in order to decrease the entropic term of the free energy in such a way that $\Delta \mathrm{G}<0$. However, when this condition holds, it is still necessary a certain amount of thermal energy to surmount the activation barrier of the reaction, $\mathrm{E}_{A}$. When this occurs, a certain amount of heat is absorbed by the reactant, so the thermal energy on its immediate surroundings is decreased. Of course, there is no such thing as the temperature of a single reacting molecule. In each sample we will have a kinetic energy distribution with a certain width. However, our assumption is to consider, as a first approximation, that all molecules have a thermal energy equal to the mean thermal energy per molecule. This is reasonable on average when the number of molecules is very big. Consequently, after a molecule has reacted and absorbed some heat, the average thermal energy of the surroundings is decreased. This perturbation expands over space and time, reducing the probability of reaction through the material. However, it 
is also expected that many molecules in the sample react at the same time, so the cooling waves may collide. The resulting scenario can be considered in an effective way as an ensemble of clusters or clouds with low probability of reaction. We define the correlation parameter $\mathrm{N}_{c}$ as the average number of surrounding molecules that cannot react on the time step after a molecule has reacted. As the reacting molecule cannot react again, when $\mathrm{N}_{c}=1$ we have a non-correlated system. But there has to be a correlation between reactions, so we expect $\mathrm{N}_{c}$ to be greater than unity, although not very big. $\mathrm{N}_{c}$ is defined as an integer (and dimensionless) quantity in its microscopic definition, but as an average and effective quantity it can be a real number.

The probability rate $\mathrm{r}_{0}$ (i.e. probability per time step $\mathrm{dt}$ ) for a single molecule to react is classically given by

$$
r_{0}=A e^{-E_{A} / k_{B} T},
$$

where $\mathrm{A}$ is the pre-exponential factor, $\mathrm{T}$ the temperature (in Kelvin degrees) and $\mathrm{k}_{B}$ the Boltzmann constant. If the molecules react in an independent way, i.e. there is no correlation, the number of reactions per time step will be proportional to the number of available molecules. Let $\mathrm{N}_{0}$ be the total amount of molecules and $\mathrm{N}$ the number of molecules that have already reacted. Then,

$$
\frac{d N}{d t}=\left(N_{0}-N\right) A e^{-E_{A} / k_{B} T}
$$

In other words, the rate of reaction is proportional to the number of available molecules multiplied by the probability rate of a single reaction. However, it is usual not to work with the number of molecules but with the occupation number $\mathrm{x}$, also called the conversion value. We define it as $\mathrm{x} \equiv N / N_{0}$, but if we work with mass in a thermogravimetric experiment we can also write

$$
x \equiv \frac{m_{0}-m}{m_{0}-m f}
$$

where $\mathrm{m}, \mathrm{m}_{0}, \mathrm{~m}_{f}$ are the instantaneous, initial and final masses of the sample, respectively. Thus we can rewrite Equation 2 as

$$
\frac{d x}{d t}=(1-x) A e^{-E_{A} / k_{B} T}
$$

This is the classical kinetic equation for uncorrelated reactions. However, we want to include the thermal correlation effect introduced above into this 
equation. In order to do this we assume as a first approximation that $\mathrm{N}_{c}$ is a constant that is specific for each material. However, it is reasonable to think that it will depend on temperature and the heat of reaction, among other factors. But we work with the constant $\mathrm{N}_{c}$ as a first approach to the problem. If there are $\mathrm{N}_{0}$ molecules to react at the beginning, we can have a maximum of $\mathrm{N}_{0} / \mathrm{N}_{c}$ clusters in the system. We impose that in each time step we can have a maximum of one reaction in each cluster. Thus the number of reactions per time step will be proportional to the number of clusters. Specifically, the overall rate will be the product of the total number of cluster and the probability rate $\mathrm{r}_{1}$ of having at least one reaction inside the cluster,

$$
\frac{d N}{d t}=\frac{N_{0}}{N_{c}} r_{1}
$$

The quantity $r_{1}$ can be factorized as the product of the probability rate of having a reaction, $\mathrm{r}_{0}$, and the probability (a purbe probability, not a rate) of having at least an available reactant in the cluster, which we call $\mathrm{P}_{1}$. We can easily find that

$$
P_{1}=1-\left(P_{2}\right)^{N_{c}},
$$

where $\mathrm{P}_{2}$ is the probability for a molecule to be in the product state, i.e. to have already reacted. Clearly, we set

$$
P_{2}=\frac{N}{N_{0}}=x .
$$

Summarizing and dividing both sides of the equation by $\mathrm{N}_{0}$ we obtain

$$
\frac{d x}{d t}=\frac{1}{N_{c}} A e^{-E_{A} / k_{B} T}\left(1-x^{N_{c}}\right),
$$

which is our main equation.

We can see that for $\mathrm{N}_{c}=1$ there is no cluster regime and all the reactions are independent. Thus the previous equation becomes Equation 4, which is again the classical uncorrelated kinetic equation. Interestingly, Equation 8 can be expanded in powers of (1-x),

$$
\frac{1}{N_{c}}\left(1-x^{N_{c}}\right)=-\frac{1}{N_{c}} \sum_{i=1}^{N_{c}}(x-1)^{i}\left(\begin{array}{c}
N_{c} \\
i
\end{array}\right) .
$$

This allows to see that as the conversion value grows, high order terms begin to vanish until the limit $\mathrm{x} \rightarrow 1$, where we recover the uncorrelated kinetics. 
This is reasonable, because at high conversion values most of the molecules in each cluster have already reacted. Notice as well that for $\mathrm{i}=1$ the expression does not depend on $\mathrm{N}_{c}$.

The limit of low $\mathrm{x}$ is even more interesting. The expansion at first order gives

$$
\left.\frac{d x}{d t}\right|_{x \ll 1} \simeq \frac{1}{N_{c}} A e^{-E_{A} / k_{B} T},
$$

where here $\mathrm{x}$ is only valid for low conversion values. This means that for an isothermal program, the initial stage of the reaction has a constant rate, as it is confirmed in [18]. This rate is the maximum achievable rate by the system, since the correlation imposes a restriction which is expressed by the correlation factor in the denominator. Moreover, if the program is nonisothermal but with a constant heating rate $\phi=\mathrm{dT} / \mathrm{dt}$, we can write

$$
\left.\frac{d x}{d T}\right|_{x \ll 1} \simeq \frac{1}{\phi N_{c}} A e^{-E_{A} / k_{B} T} .
$$

Equation 8 cannot be analytically solved, but Equation 11 has an analytical solution, giving

$$
x=\frac{A}{\phi N_{c}}\left(T e^{-E_{A} / k_{B} T}-\frac{E_{A}}{k_{B}} E_{1}\left[E_{A} / k_{B} T\right]\right), \quad x \ll 1,
$$

where $\mathrm{E}_{1}$ is the real exponential integral function. This solution can be used to describe the initial stage of the conversion curve. But notice that for increasing values of $\mathrm{N}_{c}$ the domain at which this limit is valid increases. Specifically, for $\mathrm{N}_{c} \gtrsim 2$ this regimes already covers a great part of the conversion domain. This fact makes this approximation very useful for the fit process. In Figure 1 we plot the whole integration of Equation 8 (solid line) and the direct plot of Equation 12 (dashed line) for an hypothetical case where $\mathrm{E}_{A}=4 \cdot 10^{-19}$ $\mathrm{J}, \mathrm{A}=10^{13} \mathrm{~s}^{-1}$ and $\mathrm{N}_{c}=1,2,4,8$. We see how for increasing values of $\mathrm{N}_{c}$ the divergence between the whole integration and the partial analytical solution becomes small. This is the reason why the solution for the the initial regime is very useful: almost all the conversion domain can be successfully described by the analytical expression for $\mathrm{N}_{c}$ values greater than 2 . However, the analytical solution does not saturate at $\mathrm{x}=1$ but keeps growing with temperature. In order to describe a point of inflexion and the convex final regime, we need to integrate the whole kinetic equation. We will analyze this with greater detail in the next section. 
Figure 1: Numerical integration of Equation 8 for $\mathrm{N}_{c}=1,2,4$, and 8 (solid line). Dashed lines are plots of Equation 12. All the curves have $\mathrm{E}_{A}=4$. $19^{-19} \mathrm{~J}$ and $\mathrm{A}=10^{13} \mathrm{~s}^{-1}$.

The differences between correlated and uncorrelated kinetics are noticeable. For isothermal experiments there is a tendency to keep a steady state for the initial conversion values. In general, the correlation effect introduced by $\mathrm{N}_{c}>1$ reduces the reaction rate and the shape of the curve. Specifically, the peaks shown by the derivatives exhibit an increasingly pronounced asymmetry with increasing correlation. In Figure 2 we show the effect of correlation on the overall kinetics for isothermal and non-isothermal cases. We show curves for $\mathrm{N}_{c}=1,2,4$ and 8 . Notice the strong deviations from the uncorrelated case even though the effect involves such a small size scale.

Figure 2: Comparison of uncorrelated with correlated kinetics. All curves are integrations of Equation 8. Solid, dashed, dashed-doted and dotted lines correspond to $\mathrm{N}_{c}=1,2,4$, and 8, respectively. In a) we show curves for an isothermal program at $\mathrm{T}=700^{\circ} \mathrm{C}$. In b) we show curves for a non-isothermal program with $\phi=1^{\circ} \mathrm{C} / \mathrm{min}$. $\mathrm{E}_{A}$ and $\mathrm{A}$ have the same values as in Figure 1.

\section{Experimental data and parameter fitting}

Samples were supplied by Panreac. They consist on powder of calcium hydroxide, magnesium hydroxide or calcium carbonate with high level of purity. The thermogravimetric analysis (TGA) was performed using a TGA-DTA 851e/SF/1100 from Mettler Toledo. The essays covered a temperature domain of $(30,1000)^{\circ} \mathrm{C}$ in air atmosphere with four different heating rates: $\phi=4$, 16,25 and $50^{\circ} \mathrm{C} / \mathrm{min}$. The masses of the samples were all close to $5 \mathrm{mg}$. Specifically, 15 samples were prepared with $\langle m\rangle=5.0 \pm 0.5 \mathrm{mg}$. The air flow was $50 \mathrm{ml} / \mathrm{min}$.

In this work we perform a fit process which is applied for each individual curve. With a collective fit we may encounter more accurate fits, but we are more interested in analyzing whether each curve gives consistent results or not. Furthermore, we want to see if the correlation parameter $\mathrm{N}_{c}$ can be considered as a specific constant for each solid. First, for each curve we define 
a region for the fit of the analytical expression from Equation 12. What we do is to crop the curve in a region which reasonably covers the initial stage of the reaction. In Figure 3 we show these regions in a TG curve for $\mathrm{CaCO}_{3}$ at $\phi=4^{\circ} \mathrm{C} / \mathrm{min}$ (solid line). We fit Equation 12 for the three different regions and obtain very similar results (dashed line). This means that the fit process is not very sensible on the exact choice of the region.

Figure 3: Experimental thermogravimetric data for $\mathrm{CaCO}_{3}$ with $\phi=$ $4^{\circ} \mathrm{C} / \mathrm{min}$ (solid line). The three triangles are the selected fit regions for Equation 12. The three fits give three almost identical curves (dashed).

With the initial fit we obtain values for the activation energy $\mathrm{E}_{A}$ and the ratio between the pre-exponential factor and the correlation parameter, $\mathrm{A} / \mathrm{N}_{c}$. We perform this process for each TG curve. However, as we can see in the figures, we work with thermogravimetric data, which means that the experimental output is mass versus time (or temperature). If we transform the mass into $\mathrm{x}$ using Equation 3 we perform the transformation

$$
A \rightarrow \frac{m_{0}}{m_{0}-m_{f}} A
$$

which has to be applied on Equation 8 and 12. We could let this factor to be absorbed by $\mathrm{A}$, but this would increase the variability of $\mathrm{A}$.

In order to dissociate the ratio $\mathrm{A} / \mathrm{N}_{c}$ into individual values of $\mathrm{A}$ and $\mathrm{N}_{c}$ we numerically integrate Equation 8 using the already obtained values of $\mathrm{E}_{A}$ and $\mathrm{A} / \mathrm{N}_{c}$ for increasing values of $\mathrm{N}_{c}$ until we fit the derivative curve. Although $\mathrm{N}_{c}$ can be non-integer we try only for integer values, as the fit is not intended to be very accurate but within a reasonable agreement with the experimental data.

Once we have performed the fit of the three parameters for different heating rates in a material we average the values of the activation barrier $\mathrm{E}_{A}$ which usually are the most consistent. Then we repeat all the fit process again with fixed $E_{A}$ in order to obtain more consistent results for the ratio $A / N_{c}$ and for their individual values. The obtained values are not intended to be universal for theses substances, since they strongly depend on many factors like the mass sample, the gas air flow or the type of inert atmosphere [28]. 


\section{Results and discussion}

In this section all the figures show the theoretical curves from the second step in the fit process. We begin with the fit of TG data for 4 samples of $\mathrm{Mg}(\mathrm{OH})_{2}$. In Figure 4 we can see the initial fit and the final tuning of $\mathrm{N}_{c}$ for the case $\phi=25^{\circ} \mathrm{C} / \mathrm{min}$. In the mass vs $\mathrm{T}$ plot we see how the validity of the analytical solution covers a great fraction of the conversion domain. In the plot of the mass derivative we show with a dotted line the integration for $\mathrm{N}_{c}=1$ and with a dashed line the integration for $\mathrm{N}_{c} \in[2,3]$. In general, slow heating rates give a more fluctuating derivative signal due to a finer sampling rate. These fluctuations are the reason to give an interval of values for $\mathrm{N}_{c}$. The small peak shown in the derivative plot correspond to impurities of the sample, most surely of calcium hydroxide. Next figures follow the same convention for the line styles.

Figure 4: Fit process for $\mathrm{Mg}(\mathrm{OH})_{2}$. We show a) mass versus $\mathrm{T}$ and b) mass derivative with respect to temperature versus $\mathrm{T}$ both for $\mathrm{TG}$ data in a sample heated at $\phi=25^{\circ} \mathrm{C} / \mathrm{min}$. Solid lines are always experimental. In a) we show with a dashed line the fit of Equation 12. In b) we show with a dotted line the integration of Equation 8 with $\mathrm{N}_{c}=1$, while the dashed line shows the integration for $\mathrm{N}_{c}=2$. Notice that in order to work with mass instead of $\mathrm{x}$ we have made use of Equation 3) and 13. This is also valid for Figures 5 and 6.

In Table Equation 1 we show the results for the first fit, using Equation 12. We obtain $\mathrm{E}_{A}$ and $\mathrm{A} / \mathrm{N}_{c}$ for each different heating rate. We also show the initial mass of the sample $\left(\mathrm{m}_{0}\right)$ in order to remark the importance of the fit on this experimental initial condition. Unfortunately, the fit process does not ensure that the pair $\left(\mathrm{E}_{A}, \mathrm{~A} / \mathrm{N}_{c}\right)$ is unique for a given curve. We average over $\phi=4$ and $50^{\circ} \mathrm{C} / \mathrm{min}$ cases, since we suspect that the $\phi=16^{\circ} \mathrm{C} / \mathrm{min}$ case shows a flawed curve and the $\phi=25^{\circ} \mathrm{C} / \mathrm{min}$ case has a initial mass which is too large compared with the others. We use $\left\langle\mathrm{E}_{A} / \mathrm{k}_{B}\right\rangle=22513 \mathrm{~K}$. Fixing this we can fit again the data, obtaining the results shown in Table 2. After this second process we see how the ratio $\mathrm{A} / \mathrm{N}_{c}$ gives more consistent results. Only the $\phi=25^{\circ} \mathrm{C} / \mathrm{min}$ case gives more discrepancy, in agreement with the initial mass. Furthermore we find that the values for $\mathrm{N}_{c}$ are quite consistent, always around an approximate value of 2 . 


\begin{tabular}{llll}
\hline$\phi\left({ }^{o} \mathrm{C} / \mathrm{min}\right)$ & $\mathrm{m}_{0}(\mathrm{mg})$ & $\mathrm{A} / \mathrm{N}_{c}\left(\mathrm{~s}^{-1}\right)$ & $\mathrm{E}_{A} / \mathrm{k}_{B}(\mathrm{~K})$ \\
\hline 4 & 4.8636 & $1.1049 \cdot 10^{11}$ & 21754.7 \\
16 & 4.6036 & $2.0129 \cdot 10^{9}$ & 19111.0 \\
25 & 5.5763 & $2.7303 \cdot 10^{11}$ & 22534.2 \\
50 & 4.7785 & $1.1304 \cdot 10^{12}$ & 23272.4 \\
\hline
\end{tabular}

Table 1: Table with variable values of $\mathrm{E}_{A}$ for magnesium hydroxide in air. These are the results of fitting Equation 12 in a cropped region.

On the other hand we have Passive Fire Protection (PFP), where fire hazards are reduced by processes undergone by specialized materials. These processes do not trigger further responses

\begin{tabular}{lllll}
\hline$\phi\left({ }^{\circ} \mathrm{C} / \mathrm{min}\right)$ & $\mathrm{m}_{0}(\mathrm{mg})$ & $\mathrm{m}_{f}(\mathrm{mg})$ & $\mathrm{A} / \mathrm{N}_{c}\left(\mathrm{~s}^{-1}\right)$ & $\mathrm{N}_{c}$ \\
\hline 4 & 4.8636 & 3.56 & $3.6576 \cdot 11^{6}$ & $2-3$ \\
16 & 4.6036 & 3.37 & $3.6058 \cdot 11^{6}$ & 2 \\
25 & 5.5763 & 4.08 & $2.6457 \cdot 11^{6}$ & 2 \\
50 & 4.7785 & 3.50 & $3.6951 \cdot 11^{6}$ & 2 \\
\hline
\end{tabular}

Table 2: Table with fixed $\mathrm{E}_{A}$ for magnesium hydroxide in air. These are the results from the use of Equation 12 after $\mathrm{E}_{A}$ has been fixed. Values for $\mathrm{N}_{c}$ are incremented by integer units until a fit of the derivative signal is achieved. When the derivative signal show appreciable fluctuations, an interval of $\mathrm{N}_{c}$ is given, since all the values inside the interval reasonably fit the data.

The fit process for $\mathrm{Ca}(\mathrm{OH})_{2}$ is shown in Figure 5. We show the case for $\phi=25^{\circ} \mathrm{C} / \mathrm{min}$. In the mass versus $\mathrm{T}$ plot we can see how the validity of the analytical solution covers even a wider range than the $\mathrm{Mg}(\mathrm{OH})_{2}$. This is a trace of a greater value for $\mathrm{N}_{c}$ as we will see.

In Table 3 we show the initial results for the fit process. Again, we perform an average on $\mathrm{E}$. We average using the two last heating rates, since the $\phi=4$, $16^{\circ} \mathrm{C} / \mathrm{min}$ cases have initial masses which are too far from $5 \mathrm{mg}$. We keep $\left\langle\mathrm{E}_{A} / \mathrm{k}_{B}\right\rangle=15913 \mathrm{~K}$. Fixing this we can fit again the data, obtaining the results shown in Table 4 . The results for $\mathrm{A} / \mathrm{N}_{C}$ are quite consistent again. The values of $\mathrm{N}_{c}$ are significantly greater than for the magnesium hydroxide case and the tendency is to decrease for increasing heating rates. At this point is worth recalling that the heat of reaction for the decomposition of $\mathrm{Mg}(\mathrm{OH})_{2}$ is $\simeq 1.37 \mathrm{~kJ} / \mathrm{g}$, while for $\mathrm{Ca}(\mathrm{OH})_{2}$ is approximately $1.42 \mathrm{~kJ} / \mathrm{g}[29,30]$. It is 
Figure 5: Fit process for $\mathrm{Ca}(\mathrm{OH})_{2}$. We show a) mass versus $\mathrm{T}$ and b) mass derivative with respect to temperature versus $\mathrm{T}$ both for TG data in a sample heated at $\phi=25^{\circ} \mathrm{C} / \mathrm{min}$. Solid lines are always experimental. In a) we show with a dashed line the fit of Equation 12. In b) we show with a dotted line the integration of Equation 8 with $\mathrm{N}_{c}=1$, while the dashed line shows the integration for $\mathrm{N}_{c}=5$.

reasonable to expect higher correlation effects for greater heats of reaction, since the impact of each reaction on its immediate surroundings can have more impact. However, the value of $\mathrm{N}_{c}$ may depend on other factors as well.

\begin{tabular}{llll}
\hline$\phi\left({ }^{\circ} \mathrm{C} / \mathrm{min}\right)$ & $\mathrm{m}_{0}(\mathrm{mg})$ & $\mathrm{A} / \mathrm{N}_{c}\left(\mathrm{~s}^{-1}\right)$ & $\mathrm{E}_{A} / \mathrm{k}_{B}(\mathrm{~K})$ \\
\hline 4 & 4.35568 & $9.9721 \cdot 10^{7}$ & 18185.6 \\
16 & 5.83232 & $3.7826 \cdot 10^{6}$ & 16100.2 \\
25 & 4.40393 & $2.9473 \cdot 10^{6}$ & 15694.6 \\
50 & 4.47639 & $5.9905 \cdot 10^{6}$ & 16132.2 \\
\hline
\end{tabular}

Table 3: Table with variable $\mathrm{E}_{A}$ for calcium hydroxide in air. These are the result of the first fitting process.

\begin{tabular}{lllll}
\hline$\phi\left({ }^{\circ} \mathrm{C} / \mathrm{min}\right)$ & $\mathrm{m}_{0}(\mathrm{mg})$ & $\mathrm{m}_{f}(\mathrm{mg})$ & $\mathrm{A} / \mathrm{N}_{c}\left(\mathrm{~s}^{-1}\right)$ & $\mathrm{N}_{c}$ \\
\hline 4 & 4.35568 & 3.4250 & $3.0029 \cdot 10^{6}$ & $5-10$ \\
16 & 5.83232 & 4.5230 & $2.8961 \cdot 10^{6}$ & 5 \\
25 & 4.40393 & 3.4018 & $4.0119 \cdot 10^{6}$ & 5 \\
50 & 4.47639 & 3.4501 & $4.4230 \cdot 10^{6}$ & 4 \\
\hline
\end{tabular}

Table 4: Table with fixed $\mathrm{E}_{A}$ for calcium hydroxide in air after the second fitting process.

Finally, in Figure 6 we show the $\phi=25^{\circ} \mathrm{C} /$ min case for $\mathrm{CaCO}_{3}$. In the mass versus $\mathrm{T}$ plot we can see the fit for the analytical solution. It is interesting to see that the calcium carbonate sample has some impurities, mainly of calcium hydroxide. The degradation of $\mathrm{Ca}(\mathrm{OH})_{2}$ can be easily appreciated in the figure. However, the analytical fit can also be made by cropping the 
data in such a way that we do not include the decomposition domain of the impurities.

Figure 6: Fit process for $\mathrm{CaCO}_{3}$. We show a) mass versus $\mathrm{T}$ and b) mass derivative with respect to temperature versus $\mathrm{T}$ both for TG data in a sample heated at $\phi=25^{\circ} \mathrm{C} / \mathrm{min}$. Solid lines are always experimental. In a) we show with a dashed line the fit of Equation 12. In b) we show with a dotted line the integration of Equation 8 with $\mathrm{N}_{c}=1$, while the dashed line shows the integration for $\mathrm{N}_{c}=2$.

In Table 5 we show the initial results for the fit of $\mathrm{CaCO}_{3}$ data. We have performed two experiments at $\phi=16^{\circ} \mathrm{C} / \mathrm{min}$ and three cases of $\phi=$ $50^{\circ} \mathrm{C} / \mathrm{min}$. For Table 6 we use the average value of $\mathrm{E}_{A} / \mathrm{k}_{B}=23188 \mathrm{~K}$. The second fit reveals a high consistency between the results for $\mathrm{A} / \mathrm{N}_{c}$ and again we can observe the tendency of $\mathrm{N}_{c}$ to decrease for increasing $\phi$. The heat of reaction of $\mathrm{CaCO}_{3}$ is $\simeq 1.74 \mathrm{~kJ} / \mathrm{g}[29,30]$, which keeps the expected tendency of obtaining stronger correlation for greater absorbed heat values.

These results can be applied to model and simulate the macroscopic thermal behavior of real scale samples where either one or all the three substances are added to a base material like gypsum or mortar to enhance passive fire protection. In Reference [1] we already used the results of these fits to successfully emulate the actual experimental evolution of a plaster gypsum board enriched with these three fillers.

\begin{tabular}{lllll}
\hline$\phi\left({ }^{\circ} \mathrm{C} / \mathrm{min}\right)$ & $\mathrm{m}_{0}(\mathrm{mg})$ & $\mathrm{m}_{f}(\mathrm{mg})$ & $\mathrm{E}_{A} / \mathrm{k}_{B}(\mathrm{~K})$ & $\mathrm{A} / \mathrm{N}_{c}\left(\mathrm{~s}^{-1}\right)$ \\
\hline 4 & 5.2199 & 2.974 & 23571.1 & $4.5438 \cdot 10^{6}$ \\
16 & 4.9851 & 2.855 & 23508.4 & $5.3770 \cdot 10^{6}$ \\
16 & 5.3805 & 3.077 & 23534.2 & $5.6351 \cdot 10^{6}$ \\
25 & 5.2935 & 3.051 & 22842.1 & $2.7285 \cdot 10^{6}$ \\
50 & 4.6254 & 2.655 & 23705.0 & $7.1974 \cdot 10^{6}$ \\
50 & 5.0468 & 2.903 & 23711.7 & $5.8848 \cdot 10^{6}$ \\
50 & 5.4401 & 3.129 & 22712.3 & $2.2630 \cdot 10^{6}$ \\
\hline
\end{tabular}

Table 5: Values of the parameters for variable $\mathrm{E}_{A}$ for calcium carbonate in air. 


\begin{tabular}{lllll}
\hline$\phi\left({ }^{\circ} \mathrm{C} / \mathrm{min}\right)$ & $\mathrm{m}_{0}(\mathrm{mg})$ & $\mathrm{m}_{f}(\mathrm{mg})$ & $\mathrm{A} / \mathrm{N}_{c}\left(\mathrm{~s}^{-1}\right)$ & $\left(\mathrm{N}_{c}\right)$ \\
\hline 4 & 5.22293 & 2.986 & $3.0609 \cdot 10^{6}$ & {$[10,15]$} \\
16 & 4.98703 & 2.855 & $3.9188 \cdot 10^{6}$ & 10 \\
16 & 5.39358 & 3.077 & $4.0516 \cdot 10^{6}$ & 10 \\
25 & 5.28807 & 3.051 & $3.7987 \cdot 10^{6}$ & 5 \\
50 & 4.62863 & 2.655 & $4.4048 \cdot 10^{6}$ & 5 \\
50 & 5.04985 & 2.903 & $3.5819 \cdot 10^{6}$ & 5 \\
50 & 5.43644 & 3.129 & $3.5461 \cdot 10^{6}$ & 5 \\
\hline
\end{tabular}

Table 6: Table with fixed $\mathrm{E}_{A}$ for calcium carbonate in air after the second fitting process.

\section{Conclusions}

We have seen how the proposed model gives self-consistent results. The fit process is individually applied to each curve on a first stage and then the average value of the activation barrier is taken as fixed. This improves the stability of the results for the ratio $\mathrm{A} / \mathrm{N}_{c}$. However, the values of $\mathrm{N}_{c}$ have a decreasing tendency for increasing heating rates, although for $\mathrm{Mg}(\mathrm{OH})_{2}$ is not very noticeable. This means that the approximation of considering $\mathrm{Nc}$ a constant has to be revised. Specifically, this parameter should depend on variables like heat of reaction, activation barrier or thermal energy, thus providing different values of $\mathrm{Nc}$ for different heating rates. Consequently, further work is needed to work out the actual functionality of the correlation parameter. But this first approximation reveals that the experimentally observed correlation through peak asymmetries can be explained by means of selfcooling correlations. The tendency of stronger correlations for greater heat of reaction is in qualitative agreement with the model.

The process of data fitting can be significantly improved by direct and simultaneous grid methods on the whole raw data. However, we think that for a first introduction of this correlation model it is illustrative to give a less accurate but more analytical method. The main goal was to perform a first order approach to a thermal correlation effect on the reaction rates for endothermic transitions and illustrate how this effect has to be coupled with a kinetic description where parameters are not meaningless quantities. If the self-cooling phenomenon is not considered, correlation effects are masked and classical values of $\mathrm{E}_{A}$ and $\mathrm{A}$ are misestimated. Nevertheless, the values 
of kinetic parameters have a considerable dependence on the initial mass sample, the gas air flow or the type of inert atmosphere, among other variables. This means that more precise models have to be formulated in order to provide a more realistic picture. As immediate further work, the correlation phenomenology given by $\mathrm{N}_{c}$ has to be expanded to a correlation function where temperature, activation energy or heat of reaction can be included as variables.

Our approach is a useful tool for the development of passive fire protecting materials based on the action of endothermic fillers. The kinetic model presented here is a simple way of incorporating self-cooling effects into macroscopic modeling and spatially extended simulations of materials in case of fire.

\section{Acknowledgements}

This work was supported by the Generalitat de Catalunya under Project No. 2009SGR878. We thank J.M. Chimenos and J. Formosa for their support.

\section{References}

[1] Ciudad, A; Lacasta, A. M.; Haurie, L; Formosa, J; Chimenos, J.M. Applied Thermal Engineering. 2011, 31, 3971-3978.

[2] Kontogeorgos, D; Founti, M.; Applied Thermal Engineering, 2010, 30, 1461-1469.

[3] Wullschleger, L; Wakili, K. G. Fire and Materials, 2008, 32, 103-119.

[4] Kontogeorgos, D; Mandilaras, I; Founti, M. Journal of Fire Sciences. 2011, 29, 2, 111-130.

[5] Wakili, K. G; Hugi, E. Journal of Fire Sciences. 2009, 27, 1, 27-43.

[6] Shepel, S. V.; Wakili, K. G.; Hugi, E. Numerical Heat Transfer, Part A: Applications: An International Journal of Computation and Methodology. 2010, 57, 12, 911-935.

[7] Kolaitis, D. I.; Founti, M. A. Fire Safety Journal, 2013, 58, 151-159. 
[8] Vilches, L. F.; Leiva, C.; Vale, J.; Fernandez-Pereira, C. Cement and Concrete Composites. 2005, 27, 776-781.

[9] Mehaffey, J. R.; Cuerrier, P.; Carisse, G. Fire and Materials. 1994, 18, 297-305.

[10] Borrachero, M. V.; Payá, J.; Bonilla, M.; Monzó, J. Journal of Thermal Analysis and Calorimetry. 2008, 91, 2, 503-509.

[11] Ang, C. N.; Wang, Y. C. Construction and Building Materials. 2009, $23,675-686$.

[12] Thomas, G. Fire and Materials. 2002, 26, 37-45.

[13] Kissinger, H. E. Analytical Chemistry. 1957, 29, 11, 1702-1706.

[14] Mai, D. Fourth International Symposium on Sprayed Concrete. (2002).

[15] Sultan, M. A. Fire Technology.1996, 32, 3, 239-259.

[16] Brown, M. E. Introduction to Thermal Analysis, Kluwer Academic Publishers, (2001).

[17] Elder, J. P. Thermochimica Acta 272, 1996, 272, 41-48.

[18] Brown, M. E.; Maciejewski, M.; Vyazovkin, S.; Nomen, R.; Sempere, J.; Burnham, A.; Opfermann, J.; Strey, R.; Anderson, H. L.; Kemmler, A.; Keuleers, R.; Janssens, J.; Desseyn, H. O.; Li, C. R.; Tang, T. B.; Roduit, B.; Malek, J.; Mitsuhashi, T. Thermochimica Acta. 2000, 355, 125-143.

[19] Roduit, B. Thermochimica Acta. 2000, 355, 171-180.

[20] Dickinson, C. F., Heal, G. R. Thermochimica Acta. 2009, 494, 1-14.

[21] Escardino, A.; García-Ten J.; Feliu, C.; Moreno, A. Journal of the European Ceramic Society, 2010, 30, 1989-2001.

[22] Galan, I.; Glasser, F. P.; Andrade, C. J Therm Anal Calorim, 2013, 111, 1197-1202.

[23] Halikia, I.; Neou-Syngouna, P.; Kolitsa, D. Thermochimica Acta, 1998, $320,75-88$. 
[24] L'vov, B. V.; Novichikhin, A. V.; Dyakov, A. O. Thermochimica Acta, 1998, 315, 135-143.

[25] Bhatti, A.; Dollimore, D. Thermochimica Acta, 1984, 78, 55-62.

[26] Galwey, A. K.; Laverty, G. M. Thermochimica Acta, 1993, 228, 359-378.

[27] Kissinger, H. E. Journal of Research of the National Bureau of Standars. 1956, 57,4 .

[28] Maciejewski, M. Journal of Thermal Analysis, 1992, 38, 51-70.

[29] Sha, W.; Pereira, G. B. Cement and Concrete Research. 2001, 31, 327329.

[30] Larcey, P. A.; Redfern, J. P.; Bell, G. M. Fire and Materials. 1995, 19, 283-285. 